\title{
Propiedades Psicométricas del Children's Eating Attitudes Test (ChEAT): una escala de identificación de riesgo de trastornos alimentarios en niños.
}

\author{
Luciana Soledad Elizathe ${ }^{1}$, Brenda Murawski, Fernán Guido Arana \& Guillermina \\ Rutsztein* \\ * Facultad de Psicología, Universidad de Buenos Aires, CONICET, Buenos Aires, Argentina.
}

\begin{abstract}
Resumen. El objetivo de este trabajo es presentar la adaptación y validación del Children's Eating Attitudes Test (ChEAT) en una muestra de 243 niños escolarizados de la Ciudad de Buenos Aires (CABA), de entre 9 y 13 años. Se efectuó el análisis de la estructura factorial, y se analizaron las propiedades psicométricas de la escala. Resultados: tres factores explican cerca del $40 \%$ de la varianza. El $\alpha$ de Cronbach fue de .814 para la escala total, pero disminuyó al analizar los factores por separado. La puntuación total del ChEAT se asoció con la satisfacción con la imagen corporal y la categoría de peso según índice de masa corporal. La sensibilidad fue de $73.91 \%$ y la especificidad de $72.45 \%$ para el punto de corte de 11. Finalmente, se puede concluir que el ChEAT posee buenas propiedades psicométricas y que es un instrumento promisorio para la evaluación de alteraciones en la conducta alimentaria en niños de CABA.
\end{abstract}

Palabras clave: Children's Eating Attitudes Test (ChEAT); Trastornos Alimentarios; Propiedades Psicométricas.

Title: Psychometric properties of the Children's Eating Attitudes Test (ChEAT): a measure to identify risk of eating disorders in children.

Abstract: The aim of this study is to present the adaptation and validation of the Children's Eating Attitudes Test (ChEAT) in a sample of 243 school children from the City of Buenos Aires (CABA) aged between 9 and 13. Analysis of the factorial structure was conducted, and psychometric characteristics of the scale were examined. Results: three factors explained almost $40 \%$ of the variance. Cronbach's $\alpha$ was .814 for the total scale, but declines to analyze the factors separately. ChEAT total score was related to body image satisfaction and weight category according to body mass index. Sensitivity was $73.91 \%$ and specificity $72.45 \%$ for a cut-off of 11 . Finally we can conclude that the ChEAT has adequate psychometric characteristics and that is a promising instrument for measuring disturbed eating behaviors in children of CABA. Key Words: Children’s Eating Attitudes Test (ChEAT); Eating Disorders; Psychometric Properties

\section{Introducción.}

Los trastornos alimentarios (TA) son patologías caracterizadas por una alteración alimentaria grave, que aparece asociada a una marcada insatisfacción con la imagen corporal y al temor a aumentar de peso (American Psychiatric Association [APA], 2002). Las graves consecuencias que acarrean estas patologías, y los elevados índices de mortalidad asociados (Becker, Grinspoon, Klibanski, \& Herzog, 1999; Cororve Fingeret, Warren, Cepeda-Benito, \& Gleaves, 2006; Harris \& Barraclough, 1998; Pinzon \& Beimers, 2005; Soyka, Grinspoon, Levitsky, Herzog, \& Kilbanski, 1999) han despertado el interés en el abordaje de estas

\footnotetext{
${ }^{1}$ Por favor dirigir la correspondencia relacionada con este artículo a: Lic. Luciana Soledad Elizathe, lucianaelizathe@yahoo.com.ar. Licenciada en Psicología y doctoranda de la Universidad de Buenos Aires. Becaria doctoral del CONICET.
} 
problemáticas desde hace varias décadas. Dado que estos cuadros son altamente frecuentes en adolescentes, particularmente entre las mujeres (Maganto \& Cruz, 2000; Murawski, Elizathe, \& Rutsztein, 2009; Peláez-Fernández, Labrador, \& Raich, 2007; Rutsztein, Murawski, Elizathe, \& Scappatura, 2010), los primeros trabajos que se han realizado en este campo han tomado como objeto de estudio a esta población. Sin embargo, en los últimos años se comenzó a observar el inicio a una edad más temprana. Algunos investigadores han hallado porcentajes de riesgo de TA entre el 10 y el 15\% en niños de 9 a 12 años (Bay et al., 2005; Correa, Zubarew, Silva, \& Romero, 2006; De Gracia, Marcó, \& Trujano, 2007). Otros autores (Skemp-Arlt, Rees, Mikat, \& Seebach, 2006) encontraron que un factor de riesgo para el desarrollo de TA, como lo es la insatisfacción con la propia imagen corporal, era alarmantemente habitual entre los niños de estas edades. Estos mismos autores refieren que cerca de la mitad de la muestra se encontraba insatisfecha con su propio cuerpo.

El creciente interés por el estudio de estas patologías en niños de escuelas primarias, ha llevado a algunos investigadores a desarrollar instrumentos para evaluar estos trastornos en esta población específica. Si bien actualmente existe consenso respecto a que la entrevista clínica es necesaria para determinar la presencia de esta patología (Olmsted, McFarlane, Carter, \& Trottier, 2007), resulta superior la practicidad que ofrece contar con instrumentos autoadministrables en una primera etapa, dado que permiten evaluar un gran número de sujetos simultáneamente (Garner, 2002) y son sencillos de aplicar y de puntuar (Garner \& Keiper, 2010).

Con este fin, Maloney, McGuire y Daniels (1988) realizaron una adaptación del Eating Attitudes Test (EAT) (Garner, Olmsted, Bohr, \& Garfinkel, 1982) a niños: el Children's Eating Attitudes Test (ChEAT). Este es el instrumento de mayor uso en la literatura actualizada, por lo que su adaptación y validación a niños de nuestro medio nos permitiría, además, llegar a establecer equivalencias con las investigaciones realizadas en otros países.

\section{La escala ChEAT}

Se trata de una escala que permite evaluar hábitos y actitudes relacionados con la alimentación y la imagen corporal en niños de 8 a 13 años y está conformada por tres subescalas: Dieta (que evalúa, entre otras cosas, el rechazo a consumir comida de alto contenido calórico y la preocupación por la delgadez y el peso); Control Oral, (que evalúa el control personal y social sobre la alimentación); y Bulimia y Preocupación por la comida 
(que evalúa la presencia de conductas bulímicas -atracones y vómitos- y de pensamientos persistentes en relación a la comida). Cada ítem se puede responder eligiendo entre 6 opciones de respuesta que van de siempre a nunca (escala Likert). En cada ítem (exceptuando el ítem 25 que es de puntuación inversa) la respuesta que señala mayor sintomatología es siempre (que se puntúa con el valor 3), seguida por casi siempre (que se puntúa con el valor 2), y por muchas veces (que se puntúa con el valor 1). Las tres restantes respuestas (algunas veces, casi nunca y nunca) se califican con el valor 0. El rango de puntuación del ChEAT es de 0 a 78. Maloney y colaboradores (1988) establecen que el puntaje de corte es de 20 puntos, indicando que quienes posean un puntaje igual o superior al señalado podrían estar en riesgo de presentar algún TA.

Diversos estudios han explorado las propiedades psicométricas del ChEAT hallando niveles de consistencia interna de .71 a .87 (De Gracia et al., 2007; Erickson \& Gerstle, 2007; Escoto Ponce de Leon \& Camacho Ruiz, 2008; Maloney et al., 1988; Ranzenhofer et al., 2008; Sancho, Asorey, Arija, \& Canals, 2005; Senra, Seoane, Vilas, \& Sanchezcao, 2007; Smolak \& Levine, 1994) y una confiabilidad test-retest de adecuada a buena (.56 a .84) (Escoto Ponce de Leon \& Camacho Ruiz, 2008; Maloney et al., 1988; Sancho et al., 2005). Los coeficientes de consistencia interna para cada una de las subescalas que lo forman, en cambio, fueron modestos (.57 para Control Oral, .50 para Bulimia, y .74 para Dieta) (De Gracia et al., 2007).

Con respecto a la estructura factorial del ChEAT, algunos autores han identificado soluciones de tres factores (Sinton \& Birch, 2005), de manera consistente a lo propuesto por los autores originales del test en su versión para adultos (EAT-26, Garner et al., 1982) y para niños (Maloney et al., 1988). Sin embargo la mayoría de los estudios han hallado soluciones de cuatro factores (Erickson \& Gerstle, 2007; Ranzenhofer, et al., 2008; Sancho et al., 2005; Senra et al., 2007; Smolak \& Levine, 1994) y de cinco factores (Escoto Ponce de Leon \& Camacho Ruiz, 2008; Lynch \& Eppers-Reynolds, 2005; Rojo-Moreno et al., 2011).

El objetivo de este estudio es evaluar las propiedades psicométricas del ChEAT en una muestra de niños de ambos sexos de la Ciudad Autónoma de Buenos Aires. En un trabajo anterior se realizó su adaptación lingüística y conceptual (Elizathe, Murawski, Arana, et al. 2010), como procedimiento previo necesario. A los fines del presente artículo se considerarán los siguientes objetivos: en un primer momento se analizará la capacidad discriminante de 
cada ítem de la escala. Luego, se explorará la estructura factorial de la escala y se analizará la consistencia interna de la escala total y de los factores extraídos. Asimismo, se presentarán evidencias de validez externa del ChEAT mediante la correlación de la escala total con otras variables relacionadas a los TA -satisfacción con la imagen corporal y categoría de peso según IMC- y mediante el análisis de las diferencias de medias en la escala entre sujetos con y sin riesgo de TA confirmados luego de una entrevista clínica. Finalmente, se explorará la sensibilidad y especificidad de diferentes puntos de corte del ChEAT para identificar sujetos en riesgo de TA.

\section{Método.}

\subsection{Participantes.}

Este estudio se realizó con una muestra intencional conformada por 243 niños escolarizados (122 nenas y 121 varones) de entre 9 y 13 años (Media $=11.05 ; D E=0.93$ ), que voluntariamente aceptaron participar de esta investigación. Los participantes concurren al quinto, sexto y séptimo grado de tres escuelas primarias (una pública y dos privadas) de la Ciudad Autónoma de Buenos Aires (CABA).

\subsection{Instrumentos.}

Cuestionario sociodemográfico y de sintomatología específica de trastornos alimentarios. Este cuestionario se utilizó con el fin de recabar información sobre la edad, el nivel de escolaridad, el peso actual, el peso deseado, la realización de dietas, y la percepción de la imagen corporal. Este instrumento fue diseñado ex profeso para esta investigación tomando como base el utilizado en dos Proyectos UBACyT (Programaciones 2006/2009 y 2010/2012) dirigidos por la Prof. Dra. Guillermina Rutsztein. Además, los sujetos fueron pesados y medidos por una nutricionista según las normas antropométricas de la Sociedad Argentina de Pediatría (SAP, 1986), y se calculó el Índice de Masa Corporal (IMC), un indicador que permite relacionar el peso con la altura del sujeto. En base a las curvas de IMC propuestas por el Ministerio de Salud del Gobierno de la CABA (2010), se clasificaron los sujetos de la muestra en niños/as con bajo peso, riesgo de bajo peso, normopeso, sobrepeso y obesidad.

Child/adolescent Version of the Silhouette Rating Scale (Ch/ASRS; Collins, 1991). Es una escala gráfica de siluetas que evalúa la insatisfacción con la imagen corporal. Consiste en 7 figuras de niñas y 7 de niños que conforman una graduación desde una silueta muy delgada 
hasta una que presenta un sobrepeso importante. Las respuestas se califican con una puntuación de 1 a 7 respectivamente. Cada niño debe elegir la figura que representa "cómo se ve” y la que representa “cómo le gustaría verse”. Esta escala presenta un coeficiente de consistencia interna de .71 en relación a la imagen percibida como actual y .59 en relación a la imagen corporal deseada (Collins, 1991).

The Children's Eating Attitudes Test (ChEAT, Maloney et al., 1988). La descripción de este instrumento fue realizada previamente. Se utilizó la versión local que fue adaptada lingüística y culturalmente a niños de nuestro medio (Elizathe, Murawski, Arana, et al., 2010).

Entrevista diagnóstica semiestructurada. Se trata de una entrevista individual basada en el Eating Disorder Examination (EDE, Fairburn \& Cooper, 1993). Consiste en una serie de preguntas abiertas y cerradas que contemplan los criterios para el diagnóstico de Trastorno Alimentario (Anorexia Nerviosa, Bulimia Nerviosa o Trastorno Alimentario No Especificado) explicitados en los sistemas operativos de diagnóstico vigentes, tales como el DSM-IV-TR (American Psychiatric Association, 2002) y la CIE-10 (Organización Mundial de la Salud, 1992). Esta entrevista viene siendo utilizada en el marco de los Proyectos UBACyT (Programaciones 2006/2009 y 2010/2012) dirigidos por la Prof. Dra. Guillermina Rutsztein, y se le han realizado unas pequeñas modificaciones para adaptarla a niños de escuelas primarias. Fue aplicada por psicólogas entrenadas en la detección de TA.

\subsection{Procedimiento.}

Se brindó información a las autoridades escolares y a los padres acerca del propósito del presente estudio. A su vez, teniendo en cuenta que los sujetos de la muestra son menores de edad, se solicitó el consentimiento de los padres por escrito como condición excluyente para la participación en este estudio. A los sujetos se les aseguró el carácter voluntario de su participación y la confidencialidad de los datos recabados. Se realizó un estudio de doble fase. En una primera fase, los alumnos autorizados para participar de la investigación fueron agrupados en aulas y se les entregó, previa introducción y lectura del instructivo, los cuestionarios auto-administrables que debían completar. Luego, fueron pesados y medidos por una nutricionista de acuerdo a las normas antopométricas de la SAP (1986). En una segunda fase, luego del análisis de los datos recabados, los sujetos en riesgo de TA fueron convocados y entrevistados individualmente en base a la Entrevista diagnóstica 
semiestructurada con el fin de corroborar o no la presencia de riesgo de TA y determinar el diagnóstico en caso que fuera necesario. Un número equivalente de sujetos que no presentaron riesgo también fueron entrevistados en calidad de controles, con el fin de evitar la identificación de los primeros y respetar así la confidencialidad. Los padres de los alumnos a los que se confirmó el riesgo fueron convocados a fin de derivar a esos niños a hospitales públicos para su atención.

El análisis de los datos se llevó a cabo mediante el paquete estadístico SPSS para Windows (versión 15.0). El análisis de discriminación de los reactivos se realizó para cada ítem mediante el cálculo de una prueba $t$ de diferencia de medias para muestras independientes, en función de la pertenencia al cuartil superior o inferior en la puntuación total de la escala. El análisis factorial exploratorio se basó en el método de componentes principales, utilizando la regla de Kaiser-Guttman para la identificación de los factores. Se utilizaron rotaciones ortogonales (Varimax) y un análisis paralelo de Horn (1965) para la identificación de los autovalores reales. Para el análisis de consistencia interna se calculó el coeficiente $\alpha$ de Cronbach. Por otra parte, se realizaron dos tipos deálisis distintos para aportar evidencias externas de validez. Se utilizó un cálculo de correlación bivariada de Pearson para la puntuación total del ChEAT y dos variables usualmente asociadas a los TA (satisfacción con la imagen corporal y categoría de peso según IMC). A su vez, se efectuó una prueba $t$ de diferencia de medias para grupos independientes, para explorar si existen diferencias en las medias del ChEAT en función de la pertenencia a los grupos “con riesgo de TA” y “sin riesgo de TA” luego de realizada una entrevista clínica confirmatoria. Finalmente, se utilizó la Curva ROC para identificar la sensibilidad y especificidad de la escala con diferentes puntos de corte para detectar riesgo de TA; y seleccionar el punto de corte más adecuado.

\section{Resultados.}

\subsection{Discriminación de los reactivos}

Para evaluar el poder discriminante de los reactivos se dividió la muestra en dos subgrupos en función de la pertenencia a uno de los dos cuartiles extremos de la puntuación total del ChEAT (Percentiles 25 y 75) y se compararon las medias por ítem mediante el cálculo de una prueba $t$ para muestras independientes. Se verificó que, exceptuando los ítems 21 y 25, se hallaron diferencias significativas en todos los reactivos de la escala. Los ítems 9 
y 26 (que indagan la presencia de vómitos) tuvieron una media de 0 en los dos grupos, por lo cual no se pudo efectuar la prueba. Se concluyó que la mayoría de los ítems discriminan adecuadamente entre sujetos con mayor y menor presencia de sintomatología de TA. A su vez, se decidió conservar, por el momento, los ítems que no discriminaban adecuadamente (21 y 25) para explorar su funcionamiento en la estructura factorial. Los estadísticos obtenidos en las pruebas $t$ para muestras independientes de cada reactivo pueden encontrarse en la Tabla 1.

\section{Tabla 1}

Discriminación de reactivos

\begin{tabular}{|c|c|c|c|c|}
\hline Reactivos & $\begin{array}{l}\text { Percentil } 25 \\
\text { Media (DE) }\end{array}$ & $\begin{array}{l}\text { Percentil } 75 \\
\text { Media (DE) }\end{array}$ & $\mathrm{t}$ & $p$ \\
\hline 1 & $0.08(0.32)$ & $1.34(1.27)$ & -7.617 & .000 \\
\hline 2 & $0.02(0.22)$ & 0.87 (1.17) & -5.644 & .000 \\
\hline 3 & $0.04(0.19)$ & $0.44(0.86)$ & -3.588 & .001 \\
\hline 4 & $0.00(0.00)$ & $0.23(0.64)$ & -2.786 & .007 \\
\hline 5 & $0.01(0.11)$ & $0.61(1.08)$ & -4.377 & .000 \\
\hline 6 & $0.06(0.29)$ & $0.95(1.26)$ & -5.466 & .000 \\
\hline 7 & $0.00(0.00)$ & $0.53(0.99)$ & -4.246 & .000 \\
\hline 8 & $0.08(0.36)$ & 0.47 (1.04) & -2.794 & .007 \\
\hline 9 & $0.00(0.00)$ & $0.00(0.00)$ & $\mathrm{a}$ & \\
\hline 10 & $0.01(0.11)$ & $0.18(0.56)$ & -2.297 & .025 \\
\hline 11 & $0.01(0.11)$ & $1.26(1.34)$ & -7.292 & .000 \\
\hline 12 & $0.02(0.15)$ & 1.19 (1.35) & -6.773 & .000 \\
\hline 13 & $0.02(0.15)$ & $0.56(1.08)$ & -3.908 & .000 \\
\hline 14 & $0.00(0.00)$ & 0.90 (1.28) & -5.571 & .000 \\
\hline 15 & $0.11(0.41)$ & 0.97 (1.24) & -5.239 & .000 \\
\hline 16 & $0.04(0.19$ & 0.73 (1.19) & -4.523 & .000 \\
\hline 17 & $0.01(0.11)$ & $1.16(1.27)$ & -7.106 & .000 \\
\hline 18 & $0.07(0.30)$ & 0.85 (1.16) & -5.193 & .000 \\
\hline 19 & $0.04(0.24)$ & 1.19 (1.24) & -7.250 & .000 \\
\hline 20 & $0.00(0.00)$ & $0.40(0.93)$ & -3.409 & .001 \\
\hline 21 & $0.00(0.00)$ & $0.11(0.48)$ & -1.842 & .070 \\
\hline 22 & $0.00(0.00)$ & 0.76 (1.13) & -5.300 & .000 \\
\hline 23 & $0.00(0.00)$ & $0.76(1.08)$ & -5.518 & .000 \\
\hline 24 & $0.00(0.00)$ & $0.44(0.93)$ & -3.670 & .001 \\
\hline 25 & $0.41(0.59)$ & $0.63(0.87)$ & -1.713 & .090 \\
\hline 26 & $0.00(0.00)$ & $0.00(0.00)$ & $\mathrm{a}$ & \\
\hline
\end{tabular}

Nota: a = no pudo realizarse la prueba porque las desviaciones típicas de ambos grupos son 0 .

\subsection{Análisis Factorial Exploratorio}

Se realizó un análisis de componentes principales para los 26 ítems de la escala con la muestra total $(n=243)$. Los ítems 9 y 26, que indagaban la presencia de vómitos 
autoprovocados, no pudieron ser incluidos en el análisis por presentar una media de 0 . Se repitió el procedimiento excluyendo estos ítems, conservándose 24 reactivos de la escala. El test de esfericidad de Bartlett fue satisfactorio $\left(\chi^{2}=1810.42 ; g l=276 ; p=.000\right)$ indicando así que los reactivos poseen factores en común. La medida de adecuación muestral de KaiserMeyer-Olkin fue aceptable (.759) apoyando así la utilización de estos datos en el presente análisis. Se exploró en un primer momento la solución obtenida en base al criterio de extracción de factores por raíz latente o autovalores mayores a 1, con rotación Varimax. Seguidamente se exploraron otras dos soluciones factoriales con métodos de rotación ortogonal (Varimax): forzando 3 y 4 factores. Se decidió retener los reactivos que cargaran un factor con al menos un .40, y que no cargaran doble. Finalmente, se compararon las diferentes estructuras con los modelos propuestos anteriormente con el fin de determinar cuál era la más parsimoniosa y adecuada al planteo teórico de los autores originales del test, y elegir, en función de ello, la estructura final propuesta.

\subsection{Solución inicial}

La solución inicial se obtuvo en base al criterio de extracción de factores por raíz latente o autovalores mayores a 1, con rotación Varimax. Se retuvieron así 7 factores que cumplían el criterio seleccionado, y que explicaban conjuntamente un 60.54\% de la varianza. El factor 1 explicaba la mayor parte de la varianza (14.51\%), existiendo mucha diferencia en relación a los porcentajes explicados por los últimos factores. Por otra parte, se observó que después del cuarto factor, los siguientes estaban conformados por menos de 3 ítems y éstos saturaban también en otros factores. Con el fin de determinar cuáles de estos autovalores podrían haberse obtenido por el azar, se utilizó un análisis paralelo (Horn, 1965), que es un procedimiento que permite comparar los autovalores reales con los autovalores que podrían surgir de los datos aleatorios. Este análisis permitió retener 4 factores, en tanto que el quinto, sexto y séptimo factor presentaron autovalores inferiores a los que podían surgir de los datos aleatorios.

\subsection{Solución forzando 4 factores}

A continuación, se exploró la estructura obtenida al forzar 4 factores, tal como sugería el análisis paralelo realizado en base a la solución inicial. Los factores retenidos explicaban el 46.10\% de la varianza, explicando el primer factor el mayor porcentaje (18.93\%), seguido 
por el segundo factor (11.17\%), el tercero (8.66\%) y el cuarto (7.33\%). Analizando los reactivos que integraban cada uno de estos factores se observó que el factor 1 indaga sobre aspectos vinculados a hacer dieta (restricción de algunos tipos de comida, sentimientos de culpa luego de comer y ejercicio físico para bajar de peso), pero el factor 2 también incluye reactivos vinculados a la dieta (de hecho 4 ítems saturaban para ambos factores: 2, 11, 22 y 23), aunque más focalizados en la preocupación por el cuerpo y el peso. El factor 3 está integrado por 4 ítems que corresponden a la presión social por comer, y el factor 4 por 3 ítems que evalúan preocupación por la comida (ítems que en la versión original de la escala se encontraban incluidos en el factor Bulimia y preocupación por la comida, junto con los ítems 9 y 26, excluidos del presente análisis). El ítem 25 cargó muy bajo, por lo cual fue eliminado de la estructura, conservándose 23 ítems.

\subsection{Solución forzando 3 factores}

Dado que la estructura obtenida al forzar 4 factores presentaba varios ítems que cargaban doble (en los factores 1 y 2), se repitió el análisis de componentes principales con rotación Varimax forzando 3 factores (lo cual, a su vez, coincide con la estructura propuesta por los autores originales de la escala). Se obtuvo una solución donde los 3 factores conjuntamente explicaban cerca del $40 \%$ de la varianza $(\mathrm{F} 1=22.98 \%$, $\mathrm{F} 2=8.83 \%$, y F3=7.96\%). El factor 1 (de 14 reactivos) agrupó ítems relacionados con la dieta y la preocupación por el cuerpo, que en la solución de 4 factores cargaban en el factor 1 y 2, confirmando la superposición de ambos factores. El factor 2 quedó conformado por 5 reactivos que evalúan presión social por comer y control sobre la comida. Por último, el tercer factor quedó conformado por 4 ítems que miden la preocupación por la comida. El ítem 4 -“He llegado a comer y comer sin poder parar”- no cumplió con el criterio preestablecido al saturar el factor 3 (.40) sin embargo se decidió conservarlo en esta subescala ya que alcanzó un valor muy cercano (0.35) y no cargó los otros factores. Nuevamente, el ítem 25 cargó muy bajo, por lo cual fue eliminado de la estructura, conservándose 23 ítems.

Adicionalmente, con el fin de evaluar la posible interdependencia de los factores, se probó una rotación oblicua (Oblimin directo) forzando 3 factores, arribando a resultados similares a los hallados con la rotación ortogonal, exceptuando que dos ítems cargaron doble (3 y 21) y un ítem adicional al 25 (ítem 4) cargó bajo en esta solución. 
Tabla 2

Solución factorial y consistencia interna del ChEAT

\begin{tabular}{|c|c|c|c|}
\hline & \multicolumn{3}{|c|}{$\begin{array}{c}\text { Solución Varimax } \\
3 \text { factores }\end{array}$} \\
\hline Reactivos & F1 & F2 & F3 \\
\hline 1 & 0,41 & & \\
\hline 2 & 0,62 & & \\
\hline 3 & & & 0,76 \\
\hline 4 & & & 0,35 \\
\hline 5 & 0,50 & & \\
\hline 6 & 0,46 & & \\
\hline 7 & 0,56 & & \\
\hline 8 & & 0,69 & \\
\hline 10 & 0,51 & & \\
\hline 11 & 0,77 & & \\
\hline 12 & 0,58 & & \\
\hline 13 & & 0,58 & \\
\hline 14 & 0,66 & & \\
\hline 15 & & 0,48 & \\
\hline 16 & 0,57 & & \\
\hline 17 & 0,69 & & \\
\hline 18 & & & 0,49 \\
\hline 19 & 0,62 & & \\
\hline 20 & & 0,58 & \\
\hline 21 & & & 0,61 \\
\hline 22 & 0,67 & & \\
\hline 23 & 0,79 & & \\
\hline 24 & & 0,54 & \\
\hline Autovalores & 5,52 & 2,12 & 1,91 \\
\hline (\% Varianza explicada) & $(22,98)$ & $(8,83)$ & $(7,96)$ \\
\hline \% Varianza Total explicada & & 39,77 & \\
\hline Consistencia factor & .860 & .585 & .430 \\
\hline Consistencia total & & .814 & \\
\hline Método & Compo & ipales/ & aiser \\
\hline KMO & & 0,76 & \\
\hline Prueba de Bartlett & & $(\mathrm{gl}=27$ & \\
\hline
\end{tabular}

Por último, se seleccionó la solución obtenida al forzar 3 factores con rotación Varimax, por considerársela más parsimoniosa y más coherente teóricamente. Dada la gran superposición de esta solución factorial con la estructura original propuesta por los autores de la escala, se decidió etiquetar al factor 1 como Dieta y preocupación por el cuerpo, al factor 2 como Presión social por comer, y al factor 3 como Preocupación por la comida. La solución final seleccionada se presenta en la Tabla 2. 


\subsection{Consistencia interna}

Se calculó el coeficiente $\alpha$ de Cronbach para analizar la confiabilidad de la estructura factorial propuesta (escala con 23 ítems), obteniéndose el coeficiente de .814 (n=239). Se realizó a continuación un análisis de consistencia interna para cada subescala propuesta, hallándose los siguientes valores: .860 para Dieta y preocupación por el cuerpo, .585 para Presión social por comer, y .430 para Preocupación por la comida. Los resultados obtenidos dan cuenta de un nivel adecuado de homogeneidad de la escala total y de la subescala Dieta y preocupación por el cuerpo. No obstante la consistencia es baja al analizar las otras dos subescalas por separado (Presión social por comer y Preocupación por la comida; Ver Tabla 2).

\subsection{Evidencias externas de validez}

Intentando aportar evidencias de validez concurrente para la escala compuesta por 23 ítems, se tomaron tres criterios externos. Dos de ellos (satisfacción con la imagen corporal y categoría de peso según IMC) corresponden a variables asociadas a los TA. Se efectuó un cálculo de correlación bivariada mediante el coeficiente de correlación de Pearson, verificándose correlaciones significativas pero débiles con la puntuación total en el ChEAT: satisfacción con la imagen corporal $(r=-.361, p=.000)$ y categoría de peso según IMC $(r=.369, p=.000)$. Las correlaciones indicarían que a menor satisfacción con su imagen corporal y mayor categoría de peso según su IMC, mayor riesgo de TA.

Por otra parte, se tomó una entrevista semiestructurada basada en el EDE como criterio externo y se evaluó la asociación entre el "riesgo de TA” por el ChEAT y el riesgo confirmado luego de la entrevista clínica.

Se entrevistaron en total 121 niños, de los cuales 17 presentaron una puntuación superior al punto de corte establecido por los autores originales del ChEAT $(\geq 20)$, indicando riesgo de TA. Luego de la segunda fase, se confirmó que ningún niño preseleccionado como “riesgo de TA” cumplía los criterios establecidos por el DSM-IV-TR o la CIE 10 para recibir el diagnóstico de un TA, no obstante 12 de ellos confirmaron la presencia de riesgo de TA, en función de la combinación de diferentes características reconocidas como factores de riesgo en la literatura especializada. Por otra parte, de los 104 niños que presentaron un punto inferior al punto de corte, 93 confirmaron la ausencia de riesgo luego de la entrevista, no obstante 11 presentaron finalmente riesgo de TA. 
Se realizó una prueba $t$, en donde se observaron diferencias en las puntuaciones medias del ChEAT entre los grupos con y sin riesgo de TA luego de la confirmación del EDE $(t(26,344)=4.164 ; p=.000)$, presentando una media superior en el ChEAT el grupo de los niños que confirmaron el riesgo luego de la entrevista clínica EDE (Media Riesgo=17.70; $D E=11.29$ vs Media No Riesgo=7.44; $D E=7.20$ ).

Por otra parte, se realizó una prueba Chi Cuadrado observándose una asociación entre la identificación de riesgo por el ChEAT 〔20) y la confirmación del riesgo por la entrevista EDE $(\chi 2=34.181 ; \mathrm{gl}=1 ; p=.000)$. Se evaluó dicha asociación, a su vez, considerando los puntos de corte correspondientes al percentil 80 (puntaje 11) y 85 (puntaje 13) en el ChEAT, por considerarlos relevantes para identificar población en riesgo. De esta manera se verificó que la asociación se mantenía al considerar estos otros criterios: puntaje 11 ( $\chi 2=17.303$; $\mathrm{gl}=1 ; p=.000)$, y puntaje $13(\chi 2=14.095 ; \mathrm{gl}=1 ; p=.000$; Ver Tabla 3$)$.

\section{Tabla 3}

Asociación entre riesgo/no riesgo por ChEAT (considerando diferentes puntos de corte) y riesgo/no riesgo confirmado por entrevista clínica (basada en el EDE).

\begin{tabular}{|c|c|c|c|c|c|c|}
\hline \multicolumn{7}{|c|}{$\operatorname{EDE}(n=121)$} \\
\hline $\begin{array}{l}\text { Criterio de } \\
\text { agrupación de } \\
\text { riesgo }\end{array}$ & & $\begin{array}{l}\text { Riesgo } \\
(n=23)\end{array}$ & $\begin{array}{l}\text { No riesgo } \\
\quad(n=98)\end{array}$ & $\begin{array}{c}\text { Chi } \\
\text { cuadrado }\end{array}$ & gl & $p$ \\
\hline \multirow[t]{2}{*}{ ChEAT $\geq 20$} & Riesgo (n=17) & 12 & 5 & \multirow[b]{2}{*}{34.181} & \multirow[b]{2}{*}{1} & \multirow[b]{2}{*}{.000} \\
\hline & No riesgo $(n=104)$ & 11 & 93 & & & \\
\hline \multirow[t]{2}{*}{$\operatorname{ChEAT} \geq 13$} & Riesgo (n=35) & 14 & 21 & \multirow[b]{2}{*}{14.095} & \multirow[b]{2}{*}{1} & \multirow[b]{2}{*}{.000} \\
\hline & No riesgo $(n=86)$ & 9 & 77 & & & \\
\hline \multirow[t]{2}{*}{ ChEAT $\geq 11$} & Riesgo (n=44) & 17 & 27 & \multirow{2}{*}{17.303} & \multirow{2}{*}{1} & \multirow{2}{*}{.000} \\
\hline & No riesgo $(n=77)$ & 6 & 71 & & & \\
\hline
\end{tabular}

\subsection{Sensibilidad y Especificidad con diferentes puntos de corte}

Con el objetivo de determinar cuál es el punto de corte más adecuado para identificar población en riesgo de TA, se utilizó la Curva ROC, que permite obtener los valores de sensibilidad y especificidad de la prueba al utilizar diferentes puntos de corte.

La sensibilidad de la escala al considerar el punto de corte propuesto por los autores originales del test $(\geq 20)$ (correspondiente al percentil 93 en el presente estudio) es de $52.17 \%$ y su especificidad de $94.90 \%$. Si bien la especificidad es elevada, la sensibilidad (el porcentaje de sujetos en riesgo adecuadamente identificados) es menor a la esperable para 
una prueba de screening. En cambio la sensibilidad asciende al 60.87\% al considerar como punto de corte el correspondiente al percentil $85(13)$, en tanto que, de manera esperable, la especificidad disminuye (78.57\%). Por último, se observó que la sensibilidad de la escala al considerar el punto de corte $\geq 11$ (percentil 80), era adecuada (73.91\%), y su especificidad no descendía considerablemente en relación al punto de corte anterior (72.45\%). Teniendo en cuenta que el ChEAT se trata de una herramienta de screening que se utilizará junto con una entrevista clínica en una segunda fase, se prefiere la presencia de falsos positivos que de falsos negativos, dado que esto último conllevaría la pérdida de identificación de sujetos en riesgo de TA con el consecuente peligro que entraña no intervenir precozmente en estos casos. Es por ello que se selecciona el puntaje de cộte como el más adecuado para identificar niños en riesgo de TA en nuestro contexto. Con este punto de corte son identificados correctamente un $72.72 \%$ de los niños entrevistados $(n=121)$. En la Tabla 4 se presentan los resultados obtenidos.

\section{Tabla 4}

Sensibilidad y especificidad del ChEAT (Curva ROC)

\begin{tabular}{ccccccc}
\hline $\begin{array}{c}\text { Puntos de } \\
\text { Corte }\end{array}$ & Sensibilidad & 1- Especificidad & & $\begin{array}{c}\text { Puntos de } \\
\text { Corte }\end{array}$ & Sensibilidad & 1- Especificidad \\
\cline { 1 - 2 } \cline { 5 - 6 }-1 & 1 & 1 & & & & \\
1 & 0,9565 & 0,8673 & & 17 & 0,6087 & 0,1429 \\
2 & 0,9130 & 0,7653 & & 19 & 0,6087 & 0,0816 \\
3 & 0,9130 & 0,7041 & & 20 & 0,5217 & 0,0510 \\
4 & 0,8261 & 0,6327 & & 21 & 0,4348 & 0,0408 \\
5 & 0,8261 & 0,5408 & & 22 & 0,3913 & 0,0408 \\
6 & 0,7826 & 0,4796 & & 23 & 0,3478 & 0,0306 \\
7 & 0,7391 & 0,4694 & & 24 & 0,3043 & 0,0306 \\
8 & 0,7391 & 0,4082 & & 25 & 0,2609 & 0,0306 \\
9 & 0,7391 & 0,3776 & & 27 & 0,2174 & 0,0306 \\
10 & 0,7391 & 0,3163 & & 28 & 0,1304 & 0,0306 \\
$\mathbf{1 1}$ & $\mathbf{0 , 7 3 9 1}$ & $\mathbf{0 , 2 7 5 5}$ & & 29 & 0,1304 & 0,0204 \\
12 & 0,6522 & 0,2347 & & 33 & 0,1304 & 0,0102 \\
13 & 0,6087 & 0,2143 & & 36 & 0,0870 & 0,0102 \\
14 & 0,6087 & 0,1837 & & 37 & 0,0435 & 0,0102 \\
15 & 0,6087 & 0,1531 & & 38 & 0 & 0 \\
\hline
\end{tabular}




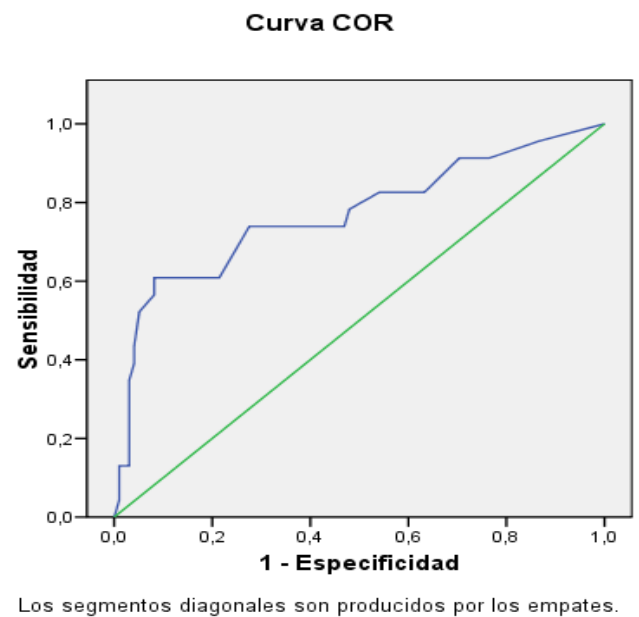

Gráfico 1: Curva ROC.

\section{Discusión.}

El objetivo de este trabajo consistió en analizar las propiedades psicométricas del ChEAT en una muestra de niños escolarizados de la CABA y completar la segunda parte de la validación de este instrumento en nuestro medio. Los resultados de este trabajo permiten concluir que esta escala tiene adecuadas propiedades psicométricas, presentando un alto grado de consistencia interna de la escala total y una estructura conformada por 3 factores que explican cerca del $40 \%$ de la varianza. Asimismo, se trata del primer estudio en nuestro medio que analiza la sensibilidad y especificidad de diferentes puntos de corte, para la detección de niños en riesgo de presentar alguna problemática alimentaria.

Los reactivos que componen la escala en la solución final discriminan adecuadamente entre sujetos con mayor y menor presencia de sintomatología de TA. La estructura final elegida, está constituida por 23 ítems, que se agrupan en 3 factores. Fueron eliminados los ítems 9, 25, y 26. El ítem 25 (Disfruto probando comidas nuevas y ricas) fue eliminado ya que no cargó ninguno de los factores; de manera similar a lo hallado en otros estudios (Ranzenhofer et al., 2008; Smolak \& Levine, 1994). Este es el único reactivo de puntuación inversa del test. Su mal funcionamiento puede deberse a que en niños de estas edades no permite discriminar entre personas con o sin alteraciones alimentarias ya que la neofobia rechazo a probar comidas nuevas y preferir lo familiar y conocido- (Katz, 2011) sería bastante habitual entre los niños de nuestro medio. Asimismo, no es del todo clara la asociación de la misma con el desarrollo de un TA (Nicolls \& Jaffa, 2007). Sancho y 
colaboradores (2005) y Senra y colaboradores (2007) eliminaron directamente este ítem antes de estudiar el funcionamiento del ChEAT en niños. Por otra parte, se propone eliminar los reactivos 9 y 26 de la solución final, ya que ninguno de los sujetos de la muestra presentó la conducta evaluada por ellos (vómitos autoinducidos). Otros autores (Sancho et al., 2005; Senra et al., 2007) también habían determinado eliminar estos ítems de la escala por considerarlos inadecuados para evaluar alteraciones alimentarias en estas edades.

En este estudio se exploraron 3 soluciones factoriales, con métodos de rotación Varimax (autovalores mayores a 1, forzando 4 factores y forzando 3 factores) y una con rotación Oblimin directo (forzando 3 factores). Si bien la solución de 4 factores se acercaba más a la estructura hallada en otros estudios, tanto en relación al porcentaje de varianza explicada como a la agrupación de los reactivos en cada factor (Sancho et al., 2005; Smolak \& Levine, 1994), en este estudio la solución de 3 factores con rotación Varimax parece ser la más parsimoniosa, dado que en ella, a diferencia de lo ocurrido con la estructura de 4 factores, los reactivos solamente saturan para uno de los factores, teniendo mayor coherencia teórica con lo planteado por los autores originales del test (Maloney et al., 1988).

El factor 1 (de 14 reactivos) agrupó ítems relacionados con la dieta y la preocupación por el cuerpo -reactivos que en su mayoría se corresponden con la subescala Dieta en la escala original (Maloney et al., 1988)-. El factor 2 quedó conformado por 5 reactivos que evalúan presión social por comer y control sobre la comida -ítems que en su mayoría coinciden con el factor Control Oral de los autores originales-. Por último, el factor 3 quedó conformado por 4 ítems que miden la preocupación por la comida -ítems que integran el factor Bulimia en la escala original-.

Al comparar esta estructura con la propuesta por Maloney y colaboradores (1988), se observa una gran similitud en el comportamiento de la mayoría de los reactivos. Se decidió etiquetar al factor 1 como Dieta y preocupación por el cuerpo, al factor 2 como Presión social por comer, y al factor 3 como Preocupación por la comida. Este último factor, llamado Bulimia en la escala original, fue re etiquetado como Preocupación por la comida, ya que los reactivos que refieren a conductas compensatorias típicas de la Bulimia Nerviosa (vómitos autoinducidos) no fueron incluidos en la solución final. Rojo-Moreno y colaboradores (2011) también observaron que estos reactivos se comportaban diferente del resto y se agrupaban en un factor etiquetado como Purga. En el presente estudio, 3 reactivos 
que integraban la subescala Control Oral en la versión original, cargaron aquí la subescala Dieta y preocupación por el cuerpo, de manera coincidente con otros trabajos: el ítem 2 “Aunque tenga hambre trato de no comer”- (Lynch \& Eppers-Reynolds, 2005; Rojo-Moreno et al., 2011; Sancho et al., 2005; Smolak \& Levine, 1994), el ítem 5 - “Corto la comida en pedacitos muy chiquititos"- (Ranzenhofer et al., 2008; Rojo-Moreno et al., 2011; Senra et al., 2007), y el ítem 19 - “Me cuido mucho en las comidas”- (Senra et al., 2007), que en varios trabajos fue eliminado de la escala por presentar una baja correlación ítem-escala y no cargar ningún factor en el análisis factorial (Escoto Ponce de Leon \& Camacho Ruiz, 2008; Sancho et al., 2005; Smolak \& Levine, 1994). Por otra parte, considerando esta subescala del test original -Control Oral-, se observó que la mayoría de los reactivos aludían a la presión social por comer y a la comparación con los demás en relación a la alimentación. Es por ello que, siguiendo la tradición de diferentes grupos de investigación que estudiaron la estructura factorial del ChEAT en niños (Escoto Ponce de Leon \& Camacho Ruiz, 2008; Lynch \& Eppers-Reynolds, 2005; Rojo-Moreno et al., 2011; Sancho et al., 2005; Senra et al., 2007) se prefirió etiquetar este factor como Presión social por comer.

Los resultados del análisis de componentes principales revelan que la estructura factorial hallada en niños argentinos es bastante similar a la propuesta en diferentes países como Estados Unidos, México y España (De Gracia et al., 2007; Erickson \& Gerstle, 2007; Escoto Ponce de Leon \& Camacho Ruiz, 2008; Lynch \& Eppers-Reynolds, 2005; Sancho et al., 2005; Senra et al., 2007; Sinton \& Birch, 2005; Smolak \& Levine, 1994; Ranzenhofer et al., 2008; Rojo-Moreno et al., 2011), explicándose las principales diferencias en el número de factores agrupados. El factor 1 de este trabajo -Dieta y preocupación por el cuerpo- coincide en las estructuras de 4 factores de Sancho y colaboradores (2005) y Senra y colaboradores (2007) con los reactivos agrupados en Miedo y preocupación por engordar y Restricción dietaria (Sancho et al., 2005) y Miedo a engordar y Restricción alimentaria (Senra et al., 2007), y en la estructura de 5 factores de Rojo-Moreno y colaboradores (2011) con Preocupación por la delgadez y Dieta. En tanto que aquellos que corresponden al factor 2 Preocupación por la comida- se agrupan en la subescala Preocupación por la comida y control oral de Rojo-Moreno y colaboradores (2011), Preocupación por la comida de Sancho y colaboradores (2005) y Deshinibición de Senra y colaboradores (2007). Los 4 ítems que se agrupan en el factor 3 -Presión social por comer-, coinciden totalmente con la subescala del mismo nombre en el modelo de Sancho y colaboradores (2005) y con la subescala Presión 
social en los modelos de Senra y colaboradores (2007) y de Rojo-Moreno y colaboradores (2011).

La escala total (23 ítems) presentó una consistencia interna elevada $(\alpha=.814)$, similar a los niveles de consistencia interna hallados en otras investigaciones (De Gracia et al., 2007; Erickson \& Gerstle, 2007; Escoto Ponce de Leon \& Camacho Ruiz, 2008; Maloney et al., 1988; Ranzenhofer et al., 2008; Sancho et al., 2005; Senra et al., 2007; Smolak \& Levine, 1994). Sin embargo, la consistencia interna de cada subescala fue sólo adecuada para el factor Dieta y preocupación por el cuerpo (Alpha=.860). Las otras subescalas presentaron índices de confiabilidad bajos (.585 para Presión social por comer, y .430 para Preocupación por la comida). Otros estudios también observaron que la confiabilidad bajaba al comparar el factor 1 con los siguientes factores (De Gracia et al., 2007; Rojo-Moreno et al., 2011). Estos resultados apoyan la sugerencia de Smolak y Levine (1994) de utilizar únicamente la puntuación total del ChEAT para identificar sujetos en riesgo, en lugar de analizar el desempeño del sujeto en cada subescala por separado. Esto último, sin embargo, podría ser útil a la hora de identificar un perfil particular de problemática alimentaria. Resultaría interesante analizar en futuros estudios la estabilidad del instrumento en un período determinado de tiempo (confiabilidad test-retest).

Por otra parte, se hallaron correlaciones significativas pero débiles entre la puntuación total del ChEAT con una medida de satisfacción con la imagen corporal y con el IMC. Esto podría comprenderse al observar la alta prevalencia de la insatisfacción con la imagen corporal entre los niños de escuelas primarias (Skemp-Arlt et al., 2006). En este sentido, otros autores también informaron correlaciones significativas pero bajas entre insatisfacción con la imagen corporal y puntuación en el ChEAT ( $r=.36$ para Smolak \& Levine, 1994; $r=.49$ para Senra et al., 2007). Por otro lado, el IMC es un indicador asociado a las problemáticas alimentarias en niños (Elizathe, Murawski, Diez, et al., 2010). De hecho, algunos estudios han sugerido que la presencia de obesidad en la infancia era tres veces más frecuente entre mujeres con Bulimia Nerviosa, que en controles (Fairburn, Welch, Doll, Davies, \& O’ Connor, 1997). Por otro lado, una revisión sobre el tema concluía que la presencia de un IMC elevado promovía factores de riesgo asociados a los TA, como ser la presión por la delgadez, la insatisfacción con la imagen corporal y la conducta dietante (Stice, 2002). Sin embargo, la baja fuerza de la correlación hallada en este estudio puede responder al hecho de que una 
persona puede sufrir una problemática alimentaria independientemente de que presente bajo peso, peso promedio o sobrepeso.

A su vez, se observó una asociación entre el "riesgo de TA” por el ChEAT y el riesgo confirmado luego de una entrevista clínica, identificándose diferencias en las puntuaciones medias del ChEAT entre los grupos con y sin riesgo de TA luego de la confirmación de la entrevista clínica. Rojo-Moreno y colaboradores (2011) también observaron diferencias entre los grupos con y sin riesgo confirmado luego de la entrevista diagnóstica y las puntuaciones del ChEAT. Estos resultados apoyan la idea de que el ChEAT discrimina adecuadamente entre niños con y sin riesgo de TA.

Por último, se presentaron diferentes puntos de corte para identificar sujetos en riesgo de TA y se analizó la sensibilidad y la especificidad de cada uno de ellos, concluyéndose que el puntaje $\geq 11$ (percentil 80) presenta una sensibilidad y una especificidad adecuada a los fines de screening, con el que fue desarrollado este test (Maloney et al., 1988). Se seleccionó un punto de corte inferior al propuesto por los autores del test $(20)$, dado que el objetivo fue adoptar un criterio que minimizara el riesgo de falsos negativos. Otros autores también propusieron bajar el punto de corte (Sancho et al., 2005). En este sentido resulta indispensable, además, la realización de una entrevista clínica que permita confirmar el TA, en una segunda etapa (Smolak \& Levine, 1994); tendencia que se puede observar en la literatura especializada de los últimos años (Bay et al., 2005; Peláez-Fernández, Labrador, \& Raich, 2008; Rodriguez-Cano, Beato-Fernandez, \& Belmonte- Llario, 2005; Rojo-Moreno et al., 2003; Rutsztein et al., 2010; Rutsztein et al., 2011).

Finalmente, se puede concluir que se cuenta con una escala adaptada, que presenta evidencias de validez para evaluar riesgo de TA en niños escolarizados de la CABA. Se espera que este avance posibilite la detección precoz de los TA con el fin de prevenir la cronicidad de los mismos y, a su vez, contribuya mediante la detección de sujetos en riesgo a la prevención del desarrollo de esta patología. Por último, se puede señalar como limitación de este estudio el haber utilizado una muestra no probabilística, con lo cual la generalización de los resultados es limitada, y se requieren mayores investigaciones sobre este tema. 


\section{Referencias.}

American Psychiatric Association. DSM-IV-TR. Versión española de la obra original en lengua inglesa Diagnostic, Statistical Manual of Mental Disorders-IV-TR publicado por la American Psychiatric Association, Washington. Masson (2002).

Bay, L., Rausch Herscovici C., Kovalskys, I., Berner, E., Orellana, L., \& Bergesio, A. (2005). Alteraciones alimentarias en niños y adolescentes argentinos que concurren al consultorio del pediatra. Archivos Argentinos de Pediatría, 103(4), 305-316.

Becker, A. E., Grinspoon, S. K., Klibanski, A., \& Herzog, D. B. (1999). Eating disorders. New England Journal of Medicine, 340, 1092-1098.

Collins, M. E. (1991). Body figure perceptions and preferences among pre-adolescent children. International Journal of Eating Disorders, 10(2), 199-208.

Cororve Fingeret, M., Warren, C. S., Cepeda-Benito, A., \& Gleaves, D. H. (2006). Eating disorder prevention research: a meta-analysis. Eating Disorder, 143, 191-213.

Correa, M., Zubarew, T., Silva, P., \& Romero, M. I. (2006). Prevalencia de riesgo de trastornos alimentarios en adolescentes mujeres escolares de la Región Metropolitana. Revista Chilena de Pediatría, 77(2), 153-160.

De Gracia, M., Marcó, M., \& Trujano, P. (2007). Factores asociados a la conducta alimentaria en preadolescentes. Psicothema, 19(4), 646-653.

Elizathe, L., Murawski, B., Arana, F. G., Diez, M., Miracco, M., \& Rutsztein, G. (2010). Detección de trastornos alimentarios en niños: Adaptación lingüística y conceptual del Children’s Eating Attitudes Test (ChEAT). Anuario de Investigaciones de la Facultad de Psicología-UBA, 17, 33-40.

Elizathe, L., Murawski, B., Diez, M., Salaberry, P., Lievendag, L., Barrios, R. M.,...Rutsztein, G. (2010). Imagen corporal y riesgo de trastornos alimentarios en niños de 9 a 12 años: diferencias en función del peso. Memorias del II Congreso Internacional de Investigación y Práctica Profesional en Psicología, XVII Jornadas de Investigación, Sexto Encuentro de Investigadores en Psicología del Mercosur. Facultad de Psicología, Universidad de Buenos Aires, 1, 44-47.

Erickson, S. J., \& Gerstle, M. (2007). Developmental considerations in measuring children's disordered eating attitudes and behaviors. Eating behaviors, 8, 224-35.

Escoto Ponce de Leon, M., \& Camacho Ruiz, E. (2008). Propiedades psicométricas del test infantil de actitudes alimentarias en una muestra mexicana. Revista Mexicana de Psicología, 25(1), 99106. 
Fairburn, C. G., \& Cooper, Z. (1993). The Eating Disorder Examination. En C. G. Fairburn \& G. T. Wilson (Eds.), Binge eating: Nature, assessment and treatment. (pp.317-360). New York: Guilford Press.

Fairburn, C. G. , Welch, S. L. , Doll, H. A., Davies, B. A. , \& O' Connor, M. E. (1997). Risk factors for bulimia nervosa. A community-based case-control study. Archives of General Psychiatry, 54, 509-17.

Garner, D. M. (2002). Measurement of eating disorder psychopathology. En: C. G. Fairburn \& K. D. Brownell (Eds.), Eating disorders and obesity: A comprehensive handbook. (pp. 141-146). New York: Guilford Press.

Garner, D. M., \& Keiper, C. D. (2010). Eating Disorders. Mexican Journal of Eating Disorders, 1, 126.

Garner D. M., Olmsted, M. P., Bohr, Y., \& Garfinkel, P. E. (1982). The Eating Attitudes Test: Psychometric features and clinical correlates. Psychological Medicine, 12, 871-878.

Harris, E. C., \& Barraclough, B. (1998) Excess mortality of mental disorder. The British Journal of Psychiatry, 173, 11-53.

Horn, J. L. (1965). A rationale and test for the numbers of factors in factor analysis. Psychometrika, 30, 179-185.

Katz, M. (2011). Comer: práctica individual, práctica social. En M. Katz, P. Aguirre, \& M. Bruera (eds.), Comer. Puentes entre la alimentación y la cultura (pp.63-109). Buenos Aires: Libros del Zorzal.

Lynch, W. C., \& Eppers-Reynolds, K. (2005). Children’s eating attitudes test: Revised factor structure for adolescent girls. Eating and Weight Disorders, 10, 222-235.

Maganto, C., \& Cruz, S. (2000). La imagen corporal y los trastornos alimenticios: una cuestión de género. Cuadernos de psiquiatría y psicoterapia del niño y del adolescente, 3(30), 45-8.

Maloney, M. J., McGuire, J. B., \& Daniels, S. R. (1988). Reliability testing of a children's version of the Eating Attitude Test. Journal of the American Academy of Child and Adolescent Psychiatry, 27, 541-543.

Ministerio de Salud del Gobierno de la Ciudad de Buenos Aires (2010). Guía Operativa 2010, Orientación para la evaluación del crecimiento. Dirección General Adjunta de Programas Centrales. Programa Nutricional.

Murawski, B., Elizathe, L., \& Rutsztein, G. (2009). Hábitos alimentarios e insatisfacción con la imagen corporal. Un estudio comparativo entre mujeres y varones estudiantes de escuelas secundarias. Anuario de Investigaciones de la Facultad de Psicología-UBA, 16, 65-72. 
Nicholls, D., \& Jaffa, T. (2007). Selective eating and other atypical eating problems. En T. Jaffa \& B. McDermott (eds.), Eating Disorders in Children and Adolescentes (pp. 144-157). New York: Cambridge University Press.

Olmsted, M. P., McFarlane, T., Carter, J., \& Trottier, K. (2007). Assessment of eating disorders. En S. Wonderlich, J. E. Mitchell, M. de Zwaan, \& H. Steiger (eds.), Annual Review of Eating Disorders Part 1. (pp. 81-100). Oxford, NY: Radcliffe Publishing.

Organización Mundial de la Salud (1992). CIE-10. Décima revisión de la clasificación internacional de las enfermedades. Madrid: Meditor.

Peláez-Fernández, M. A., Labrador, F. J., \& Raich, R. M. (2008). Comparison of single- and doublestage designs in the prevalence estimation of eating disorders in community samples. The Spanish Journal of Psychology, 11(2), 542-550.

Peláez-Fernández, M. A., Labrador, F. J., \& Raich, R. M. (2007). Prevalence of eating disorders among adolescent and young adult scholastic population in the region of Madrid (Spain). Journal of Psychosomatic Research, 62(6), 681-690.

Pinzon, J. L., \& Beimers, M. A. (2005). Medical complications in children and adolescents affected by eating disorders. British Columbia Medical Journal, 47(1), 28-34.

Ranzenhofer, L. M., Tanofsky-kraff, M., Menzie, C. M., Gustafson, J. K., Rutledge, M. S., Keil, M. F.,...Yanovski, J. (2008). Structure analysis of the Children's Eating Attitudes Test in overweight and at-risk-for-overweight children and adolescents. Eating behaviors, 9(2), 218227.

Rodriguez-Cano, T., Beato-Fernandez, L., \& Belmonte- Llario, A. (2005). New contributions to the prevalence of eating disorders in spanish adolescents: Detection of false negatives. European Psychiatry, 20(2), 173-178.

Rojo-Moreno, L., García-Miralles, I., Plumed, J., Barberá, M., Morales, M. M., Ruiz, E., \& Livianos, L. (2011). Children's eating attitudes test: Validation in a sample of Spanish schoolchildren. The International Journal of Eating Disorders. doi: 10.1002/eat.20855.

Rojo-Moreno, L., Livianos, L., Conesa, L., García-Miralles, A., Domínguez, A., Rodrigo, G.,...Vila, M. (2003). Epidemiology and risk factors of eating disorders: A two-stage epidemiologic study in a spanish population aged 12-18 years. International Journal of Eating Disorders, 34, 281-291.

Rutsztein, G., Elizathe, L., Scappatura, M. L., Murawski, B., Lievendag, L., Custodio, J., \& Galarregui, M. (2011). Imagen corporal y hábitos alimentarios en adolescentes. Un dispositivo de intervención orientado a la prevención de los trastornos alimentarios. Premio 
Facultad de Psicología (UBA): Estado actual de dispositivos e intervenciones en psicología, 33-50.

Rutsztein, G., Murawski, B., Elizathe, L., \& Scappatura, M. L. (2010). Trastornos alimentarios: Detección en adolescentes mujeres y varones de Buenos Aires. Un estudio de doble fase. Revista Mexicana de Trastornos Alimentarios, 1, 48-61.

Sancho, C., Asorey, O., Arija, V., \& Canals, J. (2005). Psychometric characteristics of the Children's Eating Attitudes Test in a Spanish sample. European Eating Disorders Review, 13(5), 338343.

Senra, C., Seoane, G., Vilas, V., \& Sanchezcao, E. (2007). Comparison of 10- to 12-year-old boys and girls using a Spanish version of the children's eating attitudes test. Personality and Individual Differences, 42(6), 947-957.

Sinton, M., \& Birch, M. S. (2005). Weight status and psychosocial factors predict the emergence of dieting in preadolescent girls. International Journal of Eating Disorders, 38(4), 346-354.

Skemp-Arlt, K. M., Rees, K. S., Mikat, R. P., \& Seebach, E. E. (2006). Body Image Dissatisfaction Among Third, Fourth, and Fifth Grade Children. Californian Journal of Health Promotion, 4(3), 58-67.

Smolak, L., \& Levine, M. P. (1994). Psychometric properties of the children's eating attitudes test. International Journal of Eating Disorders, 16(3), 275-282.

Sociedad Argentina de Pediatría (1986). Criterios de diagnóstico y tratamiento. Crecimiento y desarrollo. Buenos Aires: Roemmers.

Soyka, L. A., Grinspoon, S., Levitsky, L. L., Herzog, D. B., \& Kilbanski, A. (1999). The effects of anorexia nervosa on bone metabolism in female adolescents. Journal of Clinical Endocrinology and Metabolism, 84, 3389-4496.

Stice, E. (2002). Risk and maintenance factors for eating pathology: A meta-analytic review. Psychological Bulletin, 128(5), 825-848. 